\title{
Lidil
}

Revue de linguistique et de didactique des langues

$57 \mid 2018$

Démarches créatives, détours artistiques et appropriation des langues

\section{Marie-Christine Pollet et Carole Glorieux (dir.), Argumenter dans les écrits scientifiques}

Presses universitaires de Namur, 2016, 222 p.

\section{Tatiana Aleksandrova}

\section{OpenEdition \\ Journals}

Édition électronique

URL : http://journals.openedition.org/lidil/4847

DOI : 10.4000/lidil.4847

ISSN : $1960-6052$

Éditeur

UGA Éditions/Université Grenoble Alpes

Édition imprimée

ISBN : 978-2-37747-048-8

ISSN : $1146-6480$

Référence électronique

Tatiana Aleksandrova, « Marie-Christine Pollet et Carole Glorieux (dir.), Argumenter dans les écrits scientifiques », Lidil [En ligne], 57 | 2018, mis en ligne le 01 mai 2018, consulté le 25 septembre 2020. URL : http://journals.openedition.org/lidil/4847 ; DOI : https://doi.org/10.4000/lidil.4847

Ce document a été généré automatiquement le 25 septembre 2020

(C) Lidil 


\title{
Marie-Christine Pollet et Carole Glorieux (dir.), Argumenter dans les écrits scientifiques
}

Presses universitaires de Namur, 2016, 222 p.

\author{
Tatiana Aleksandrova
}

\section{RÉFÉRENCE}

Marie-Christine Pollet et Carole Glorieux (dir.), Argumenter dans les écrits scientifiques, Presses universitaires de Namur, 2016, 222 p.

1 Cet ouvrage regroupe 15 contributions qui traitent la thématique de l'argumentation dans les écrits scientifiques. Deux dimensions réunissent ces études: la réflexion autour de la notion des littéracies universitaires et celle de la didactique de cette même discipline.

2 Après un historique du domaine des littéracies académiques et une description de sa place en France et en Europe, trois contributions abordent les notions d'argumentation, de subjectivité et d'image dans les textes scientifiques. L'article de Christian Plantin discute la notion d'argumentation en sciences humaines et sciences dures, en montrant qu'il ne s'agit pas de deux pratiques opposées mais d'une pratique sociale propre à toutes les disciplines, même si en sciences dures l'argumentation consiste davantage à faire une démonstration et à en présenter les preuves. L'auteur discute les notions de type d'argument et d'argumentation basée sur la causalité. La contribution proposée par Alain Rabatel discute la notion de subjectivité dans l'argumentation. Il montre qu'au-delà de l'utilisation des pronoms personnels, les moyens de son expression sont nombreux et souvent indirects. Seule la prise en compte du contexte énonciatif permet d'interpréter le degré de subjectivité exprimée par l'auteur. Maria Giulia Dondero propose une discussion autour de la notion de rhétorique visuelle. Elle étudie le rôle de 
l'image et notamment de la photographie dans les textes scientifiques qui portent sur le phénomène physique des trous noirs.

3 Les articles suivants réfléchissent sur la didactique des écrits académiques. Le travail de Caroline Scheepers et Stéphanie Delneste analyse les réponses à un questionnaire sur les pratiques de rédaction, adressé à des scripteurs confirmés et novices. La place du scripteur, les notions de subjectivité et de neutralité y sont discutées. La contribution de Christiane Donahue décrit et analyse l'assessement, moyen d'évaluation des pratiques écrites largement utilisé dans les universités aux États-Unis. Après la présentation des dispositifs existant dans ces universités, l'auteure propose une évaluation du dispositif, en discutant la difficulté des étudiants à relier les connaissances acquises dans les formations diverses pour en faire une compétence globale susceptible d'en faire des scripteurs compétents. Francis Grossmann étudie les marqueurs lexicaux associés à l'évidence dans un corpus de textes produits par des étudiants et des doctorants. Il identifie quelques structures syntaxiques et expressions utilisées par les scripteurs, tout en évaluant leur fréquence et leur place dans le texte.

4 Fanny Rinck discute la notion de polyphonie et la place de l'auteur dans le discours scientifique. Pour sa part, Carole Glorieux analyse les pratiques rédactionnelles en les mettant en rapport direct avec un genre particulier qui est le mémoire d'application réalisé par les étudiants de la faculté de journalisme. L'obligation de réunir dans un seul travail plusieurs genres d'écrits : écrit scientifique, discours professionnel et le discours d'application pratique, semble poser de grandes difficultés aux étudiants. Roselyne Lampron et Christiane Blaser proposent une réflexion sur la notion de genre du mémoire professionnel et de la place de l'auteur dans cet écrit. Elles traitent particulièrement des notions comme "légitimité " et "crédibilité », et lancent quelques pistes de réflexions didactiques autour de cette question. L'article d'Odette Gagnon évoque la notion d'arrimage des énoncés dans le texte, en analysant les procédés de cohésion dans les travaux d'étudiants. Quant à la contribution de Catherine Gravet, elle aborde plusieurs éléments des écrits scientifiques tels que l'utilisation des pronoms personnels, les notes de bas de page et la présentation des références bibliographiques. Victor Ferry et Emmanuelle Danblon discutent la notion de la rhétorique et notamment la rhétorique de la critique.

5 Le recueil se termine par la contribution d'Isabelle Delcambre focalisée sur les apports de toutes ces études à la didactique des littéracies avancées. L'auteure évoque la richesse des données et félicite les tentatives des chercheurs à trouver des applications pratiques pour essayer de résoudre les difficultés des apprenants. Elle mentionne néanmoins qu'il reste encore beaucoup à faire pour relier la recherche à la pratique. Cet ouvrage, riche en apports théoriques et approches concrètes aux problèmes liés aux écrits académiques, permet au lecteur d'avoir une vision sur les avancées de la recherche en ce domaine. 


\section{AUTEURS}

TATIANA ALEKSANDROVA

LIDILEM, Université Grenoble Alpes 